\title{
A Study on the Impact of Surgical Duration on Esophageal Cancer
}

\author{
Makoto Kobayashi \\ Eiji Yoshida \\ Takuro Kyuno \\ Ryoko Kawagishi \\ Kei Sato \\ Tsuyoshi Kono \\ Takehiro Chiba \\ Hitoshi Yonezawa \\ Osamu Funato \\ Akinori Takagane
}

Department of Surgery, Hakodate Goryoukaku Hospital, Hakodate City, Hokkaido, 040-86II, Japan
Correspondence: Makoto Kobayashi Department of Surgery, Hakodate Goryoukaku Hospital, 38-3 Goryoukakucho, Hakodate City, Hokkaido, 040-86I I, Japan

Tel +8I- |38-5I-2295

Fax+8I-138-56-2696

Email koba86gg@gmail.com
Purpose: Faster surgery has been associated with better outcomes. In the present study, we aimed to determine whether surgical duration affected overall survival (OS) in patients undergoing esophagectomy. Additionally, we assessed factors that influence surgical duration.

Patients and Methods: This single-center retrospective cohort study included 128 patients who underwent Ivor-Lewis esophagectomy by a single surgeon between 2005 and 2019. The Cox proportional hazard model was used to evaluate the association of OS with the following variables: surgical duration, neo-adjuvant chemotherapy, pathological grade of depth of tumor invasion, lymph node (LN) metastasis, body mass index (BMI), and cervical LN dissection. Additionally, factors associated with prolonged surgical duration were examined by logistic regression analysis.

Results: Based on the multivariate analysis, surgical duration was not associated with OS [hazard ratio (HR), 1.065; 95\% confidence interval (CI), 0.586-1.937; $P=0.837$ ]. On the other hand, tumor invasion (HR, 2.901; 95\% CI, 1.483-5.674; $P=0.002)$ and LN metastasis (HR, 2.338 2.403; 95\% CI, 1.237-4.420 1.257-4.593; $P=0.0090 .008$ ) significantly influenced OS. The assessment of variables affecting surgical duration showed that BMI had a significant effect on surgical duration (odds ratio, 2.790; 95\% CI, 1.254-6.204, $P=0.012$ ).

Conclusion: According to the analysis of patients who underwent the same surgical approach by a single surgeon, surgical duration of esophagectomy for esophageal cancer was significantly influenced by BMI; however, surgical duration had no impact on patient survival.

Keywords: esophagectomy, Ivor-Lewis approach, depth of tumor invasion, lymph node metastasis, body mass index, overall survival

\section{Introduction}

Recently published data suggest that many risk factors are involved in the occurrence of postoperative complications, including the impact of surgical duration among patients undergoing pancreatoduodenectomy, biliary procedures, and esophageal operations. ${ }^{1-3}$ It is difficult to determine whether differences in surgical duration affect postoperative survival because surgical duration may be affected by various factors, such as a difference in the surgical approach, difference in the skill of individual surgeons, and difference in the stage of cancer progression. In the present study, we aimed to evaluate the relationship between surgical duration and overall survival (OS) and subsequently determined the factors associated with surgical duration in cases operated by a single surgeon using the same surgical approach. 


\section{Graphical Abstract}

\section{A study on the impact of surgical duration on esophageal cancer}

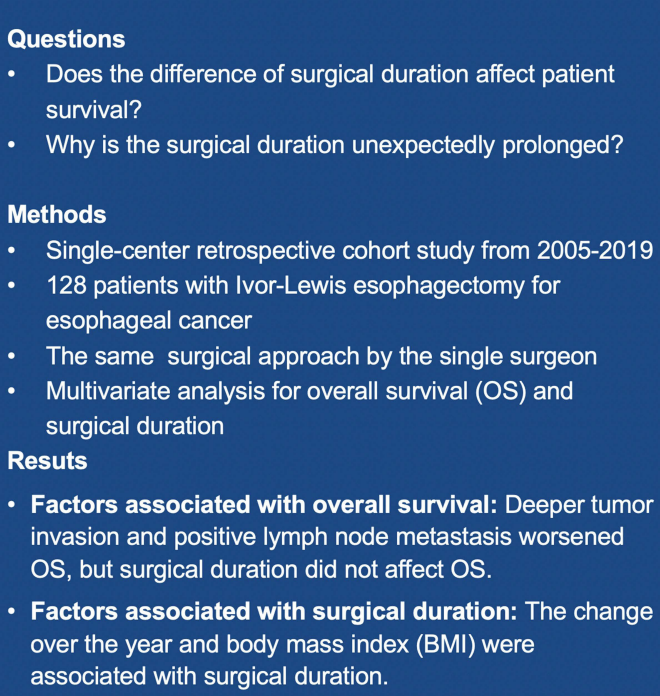

Kobayashi M, et al. Open Access Surgery 2021

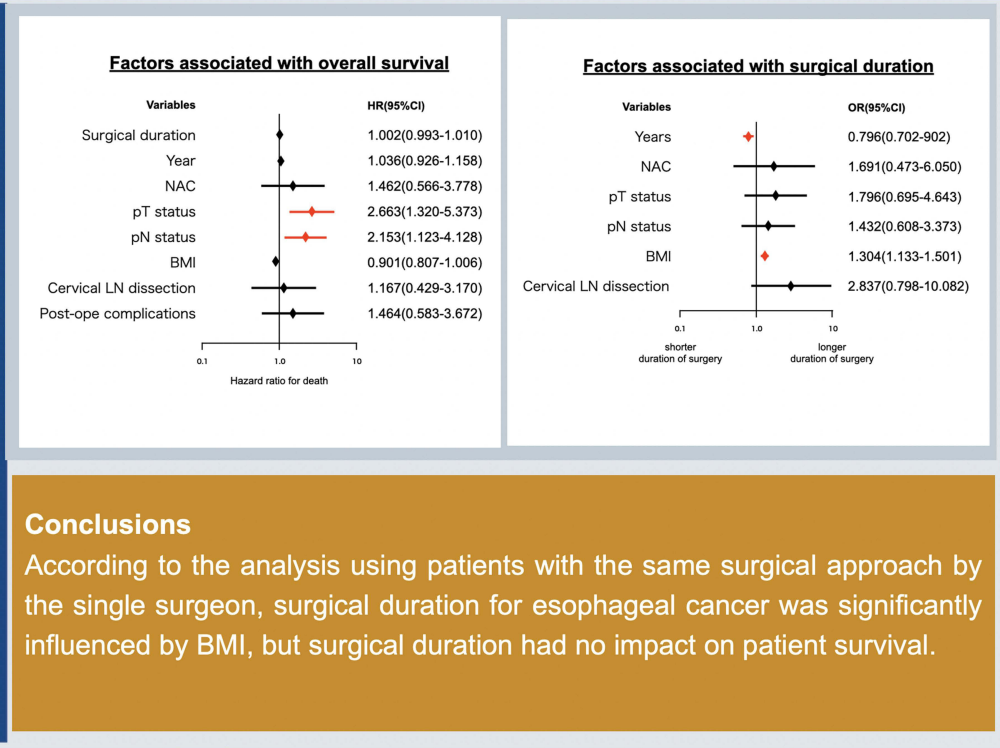

Conclusions

According to the analysis using patients with the same surgical approach by the single surgeon, surgical duration for esophageal cancer was significantly influenced by BMI, but surgical duration had no impact on patient survival.

\section{Patients and Methods}

\section{Patient Selection}

This was a retrospective cohort study of consecutive patients who underwent esophagectomy for esophageal cancer in Hakodate Goryoukaku Hospital between January 2005 and December 2019. One hundred and twenty-eight patients who received right thoracotomy and laparotomy (Ivor-Lewis esophagectomy) for a curative resection were selected, and their data were provided to this study. Cases where lymph node (LN) dissection of the mediastinum was not performed were excluded. All patients enrolled in the study underwent surgery by a single surgeon with a board certification from the Japan Esophageal Society. The study protocol was approved by the ethics committee of Hakodate Goryoukaku Hospital (Approval No. 2020-077). Informed consent was waived because of the retrospective study design; however, the committee also verified that the data remained confidential by concealing the privacy of participants and complying with the Declaration of Helsinki.

\section{Definitions}

The cancer status of patients was categorized based on the TNM Classification of Malignant Tumors, 7 th edition. ${ }^{4}$
Indications for surgical treatment and neo-adjuvant chemotherapy (NAC) were determined according to the Guidelines for Diagnosis and Treatment of Carcinoma of the Esophagus. ${ }^{5}$ The Clavien-Dindo classification was used to grade postoperative complications. ${ }^{6}$ Body mass index (BMI; $\mathrm{kg} / \mathrm{m}^{2}$ ) was calculated based on the direct measurement of height and weight at surgery. The definition of surgical duration indicates the time in which the surgical procedure was actually performed, excluding the anesthesia time during pre- and post-surgical operations.

\section{Patient Management}

Based on the proposal of a Japan Clinical Oncology Group Study (JCOG9907), the NAC regimen included cisdiamine platinum and 5-fluorouracil, which were administered twice before surgery to the candidates of preoperative treatment. ${ }^{7}$ All patients were scheduled to undergo Ivor-Lewis esophagectomy with extended lymphadenectomy. To briefly explain the surgical procedure, skin incisions were created for the upper abdominal laparotomy and right thoracotomy. After resecting the thoracic esophagus with cancer and dissecting the mediastinal $\mathrm{LN}$, right intrathoracic anastomosis was created in the thorax 
between the remnants of the esophagus and the gastric conduit using a circular anastomosis device. Cervical LN dissection was also performed in the neck region when the preoperative computed tomography (CT) imaging detected enlarged cervical LN or the site of the tumor was in the upper part of the esophagus. Postoperative management was performed according to our previously described protocol. 8 Postoperative adjuvant chemotherapy was indicated and administered in patients with positive pathological LN metastasis based on the results of a Japanese Clinical Oncology Group Study (JCOG9204). ${ }^{10}$ Patients were evaluated at 6-month intervals after surgery, and the follow-up included physical examination, measurement of serum tumor markers, and thoraco-abdominal CT scans. Esophago-gastro-duodenoscopy was performed every three years. Positron-emission tomography was used in patients with unexpected abnormalities in serum tumor markers and in those with suspicious cancer recurrence by CT scans. Patient survival was defined as the time to death from any cause. Final confirmation of patient's life and death was made in June 2021. The minimum period of observation was one year and six months.

\section{Data Management for Multivariate Analysis}

Baseline data were acquired from electronic medical records. For multivariate analysis of surgical duration, some variables were categorized into two groups as follows: Based on the pathologic depth of tumor invasion (pT), the patients were divided into the pT1-2 and pT3-4a groups; Based on the pathological grade of LN metastasis $(\mathrm{pN})$, the patients were divided into the $\mathrm{pN} 0$ and $\mathrm{pN} 1-3$ groups. The occurrence of postoperative complications was defined according to the Clavien-Dindo classification when it showed a grade of II or higher.

\section{Statistical Analysis}

Surgical duration of each categorized group was presented as means with standard deviations and compared by Student's $t$-test for univariate analysis. Pearson analysis was used to analyze the correlation of surgical duration with two variables, BMI and year, in which esophagectomy was performed. The Kaplan-Meier method was used to estimate survival rates, and comparisons were performed using the Log rank test. To assess the association of surgical duration with patient survival, OS was examined using the Cox proportional hazard models with the following variables: surgical duration, presence of NAC, pT status, $\mathrm{pN}$ status, BMI, and implementation of cervical LN dissection. Logistic regression models were used to assess the relationship between the difference in surgical duration and the following variables: years, NAC, pT status, $\mathrm{pN}$ status, BMI, and cervical LN dissection. Hazard ratios (HRs), odds ratios (ORs), and 95\% confidence intervals (CIs) were determined for all variables. In all analyses, a $P$-value of $<0.05$ indicated statistical significance. All statistical analyses were performed using IBM SPSS Statistics for Macintosh, version 21.0 (IBM, Armonk, NY).

\section{Results}

\section{Patient Characteristics}

All patient characteristics are presented in Table 1. The subjects of this study were all Japanese. Figure 1 shows the estimated survival rate after esophagectomy in 128 cases with the Ivor-Lewis approach (3-year OS: 68.2\%, 5-year OS: 63.5\%).

\section{Factors Associated with OS}

Regarding patient survival analyzed by Log rank test, the group with deeper tumor invasion and the group with positive LN metastasis showed significant lower survival rates (Table 2). The Cox proportional-hazards models (Figure 2) showed that the groups with deeper tumor invasion (OR 2.663, $P=0.002$ ) and positive $\mathrm{LN}$ metastasis (OR 2.153, $P=0.008$ ) had significantly lower OS. In contrast, surgical duration did not affect OS $(P=0.837)$.

\section{Factors Associated with Surgical Duration}

Results of the univariate analysis are shown in Table 2. Surgical duration was significantly longer in the group with positive LN metastasis and the group that underwent additional cervical LN dissection. Pearson correlation analysis revealed that there was a significant positive correlation between surgical duration and BMI $(\mathrm{r}=0.196, P=$ 0.027). Change of year also had a significant negative correlation with surgical duration $(\mathrm{r}=-0.260, P=$ 0.003). Assessing the variables affecting surgical duration (Figure 3), the logistic regression analysis revealed that BMI (OR, 1.304; $P=0.012$ ) and the changes over the years (OR, 0.796; $P=0.000)$ were significantly associated with surgical duration. 
Table I Patients' Characteristics

\begin{tabular}{|c|c|}
\hline Parameter & Data \\
\hline Patients, $\mathrm{n}$ & 128 \\
\hline Age, mean (SD) & $68(8.3)$ \\
\hline Sex (male), n (\%) & $104(8 I)$ \\
\hline BMI, mean (SD) & $21.6(3.5)$ \\
\hline Neo-adjuvant chemotherapy, n (\%) & $43(34)$ \\
\hline $\begin{array}{l}\text { Surgical duration }(\mathrm{min}) \text {, mean } \\
(\mathrm{SD}) / \text { median }\end{array}$ & $236(40) / 237$ \\
\hline Blood loss (gm), mean (SD) & $148(162)$ \\
\hline Pathological diagnosis, $\mathrm{n}$ & $\begin{array}{l}\text { Squamous cell carcinoma } \\
\text { II2 } \\
\text { Adenocarcinoma II } \\
\text { Neuroendocrine } \\
\text { carcinoma } 3 \\
\text { Basaloid carcinoma I } \\
\text { Carcinosarcoma I }\end{array}$ \\
\hline Tumor location, $\mathrm{n}$ & Ut 6/Mt 64/Lt 47/Ae II \\
\hline 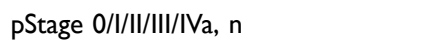 & $20 / 28 / 29 / 37 / 14$ \\
\hline PT 0/I/2/3/4a, n & $5 / 55 / 16 / 42 / 10$ \\
\hline $\mathrm{pN} \mathrm{0/I/2/3,n}$ & $63 / 34 / 20 / 11$ \\
\hline $\begin{array}{l}\text { Number of harvested lymph node, } \\
\text { mean (SD) }\end{array}$ & $38(18)$ \\
\hline Post-operative complications, n (\%) & II (8.6) \\
\hline Pneumonia & $7(5.5)$ \\
\hline Pyothorax & $3(2.3)$ \\
\hline Recurrent nerve palsy & $2(1.6)$ \\
\hline Anastomotic leak & $\mathrm{I}(0.8)$ \\
\hline In-hospital death, n (\%) & $2(1.6)$ \\
\hline $\begin{array}{l}\text { In-hospital days after surgery, median } \\
\text { (range) }\end{array}$ & $15(12-97)$ \\
\hline
\end{tabular}

Abbreviations: SD, standard deviation; BMI, body mass index; Ut, upper thoracic esophagus; Mt, middle thoracic esophagus; Lt, lower thoracic esophagus; Ae, abdominal esophagus; pStage, post-surgical pathological staging; $\mathrm{pT}$, pathological grades of depth of tumor invasion; $\mathrm{pN}$, pathological grades of lymph node metastasis.

\section{Discussion}

In the present study, we evaluated the association of surgical duration with OS in patients undergoing Ivor-Lewis esophagectomy, which was performed by a single surgeon. Our analyses revealed that surgical duration was not associated with patient survival.

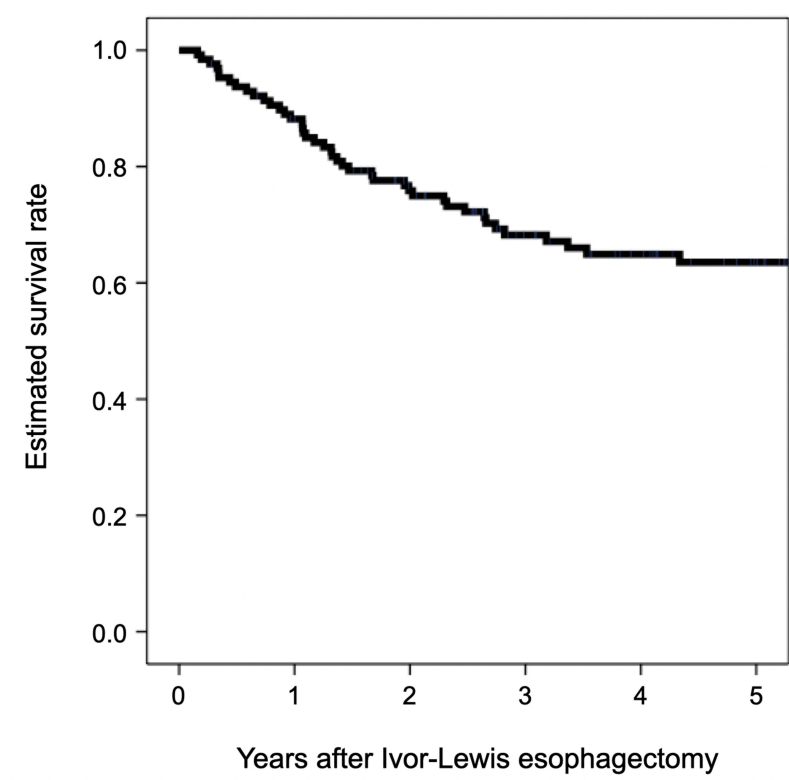

Figure I Five-year overall survival after esophagectomy in 128 cases with IvorLewis approach.

Longer surgical duration was previously reported to be associated with a greater amount of bleeding and higher incidence of postoperative complications. A retrospective cohort study that enrolled 2651 patients who underwent IvorLewis esophagectomy reported that prolonged surgical duration had an independent adverse impact on postoperative complications but not on 30-day mortality. ${ }^{11}$ This study, which involved patients undergoing Ivor-Lewis esophagectomy, also revealed that surgical duration did not affect OS (HR 1.065, $\mathrm{P}=0.837$ ), but preoperative lower BMI could be associated with lower OS (HR 0.901, $P=0.058$ ). On weight loss and postoperative prognosis, a study of 922 patients with esophageal cancer from the Netherlands concluded that patients who had a weight loss of $10 \%$ or more had increased perioperative risk and decreased 5-year survival. ${ }^{12}$ Another report from China assessed the impact of BMI in 2031 patients that underwent esophagectomy and reported that high BMI $\left(\mathrm{BMI}>23 \mathrm{~kg} / \mathrm{m}^{2}\right)$ had a significant impact on postoperative complications but improved 10-year OS. ${ }^{13}$

What are the factors that cause prolonged surgical duration? In order to identify factors associated with differences in surgical duration, several explanatory variables should be considered as potential biases, with surgeon's experience and surgical skill level among the first. To eliminate these biases, we targeted patients who underwent surgery using the same 
Table 2 Univariate Analysis for Surgical Duration and Overall Survival

\begin{tabular}{|c|c|c|c|c|c|}
\hline Variables & Group, $\mathbf{n}$ & $\begin{array}{l}\text { Surgical Duration, Mean } \pm \\
\text { SD min }\end{array}$ & $\begin{array}{l}\text { Student's } t \text {-Test, } \\
P \text { value }\end{array}$ & $\begin{array}{l}\text { 3-y OS, } \\
5-y \text { OS }\end{array}$ & $\begin{array}{l}\text { Log Rank Test, } \\
P \text { value }\end{array}$ \\
\hline NAC & $\begin{array}{l}\text { Yes, } n=43 \\
\text { No, } n=85\end{array}$ & $\begin{array}{l}241 \pm 36 \min \\
234 \pm 42 \min \end{array}$ & 0.324 & $\begin{array}{l}63.4 \%, 63.4 \% \\
70.4 \%, 64.0 \%\end{array}$ & 0.647 \\
\hline pT status & $\begin{array}{l}\text { PT } 1-2, n=76 \\
\text { PT } 3-4, n=52\end{array}$ & $\begin{array}{l}231 \pm 37 \mathrm{~min} \\
244 \pm 43 \mathrm{~min}\end{array}$ & 0.088 & $\begin{array}{l}85.4 \%, 79.8 \% \\
42.8 \%, 37.5 \%\end{array}$ & 0.000 \\
\hline $\mathrm{pN}$ status & $\begin{array}{l}\mathrm{pN}(-), n=63 \\
\mathrm{pN}(+), n=65\end{array}$ & $\begin{array}{l}229 \pm 38 \min \\
243 \pm 42 \min \end{array}$ & 0.046 & $\begin{array}{l}86.4 \%, 79.0 \% \\
50.8 \%, 48.0 \%\end{array}$ & 0.001 \\
\hline Cervical LN dissection & $\begin{array}{l}\text { Yes, } n=45 \\
\text { No, } n=83\end{array}$ & $\begin{array}{l}248 \pm 32 \min \\
230 \pm 43 \min \end{array}$ & 0.011 & $\begin{array}{l}62.8 \%, 62.8 \% \\
71.2 \%, 64.7 \%\end{array}$ & 0.488 \\
\hline $\begin{array}{l}\text { Post-operative } \\
\text { complications* }\end{array}$ & $\begin{array}{l}\text { Occurred, } n=11 \\
\text { No, } n=117\end{array}$ & $\begin{array}{l}243 \pm 54 \mathrm{~min} \\
236 \pm 39 \mathrm{~min}\end{array}$ & 0.557 & $\begin{array}{l}6.4 \%, 36.4 \% \\
71.8 \%, 66.5 \%\end{array}$ & 0.055 \\
\hline
\end{tabular}

Note: *The Clavien-Dindo classification showed grade II or higher.

Abbreviations: NAC, neo-adjuvant chemotherapy; pT, pathological grades of depth of tumor invasion; pN, pathological grades of lymph node metastasis; LN, lymph node; $\mathrm{SD}$, standard deviation; OS, overall survival.

approach by a single surgeon. Second, the progression of cancer tends to make resection difficult, which should be considered in determining the variables that affect surgical duration. In patients with more invasive cancer and in those with positive $\mathrm{LN}$ metastasis, the procedure time, which may be required for tumor excision and definitive LN dissection, is expected to be longer. However, in our multivariate analysis, its influence was not statistically significant. Third, increased intra-abdominal fat is likely to prolong the surgical procedure. In the present study, logistic regression analysis revealed that high BMI was significantly associated with longer surgical duration (OR 1.304, $P=0.000)$. Fourth, the present analysis

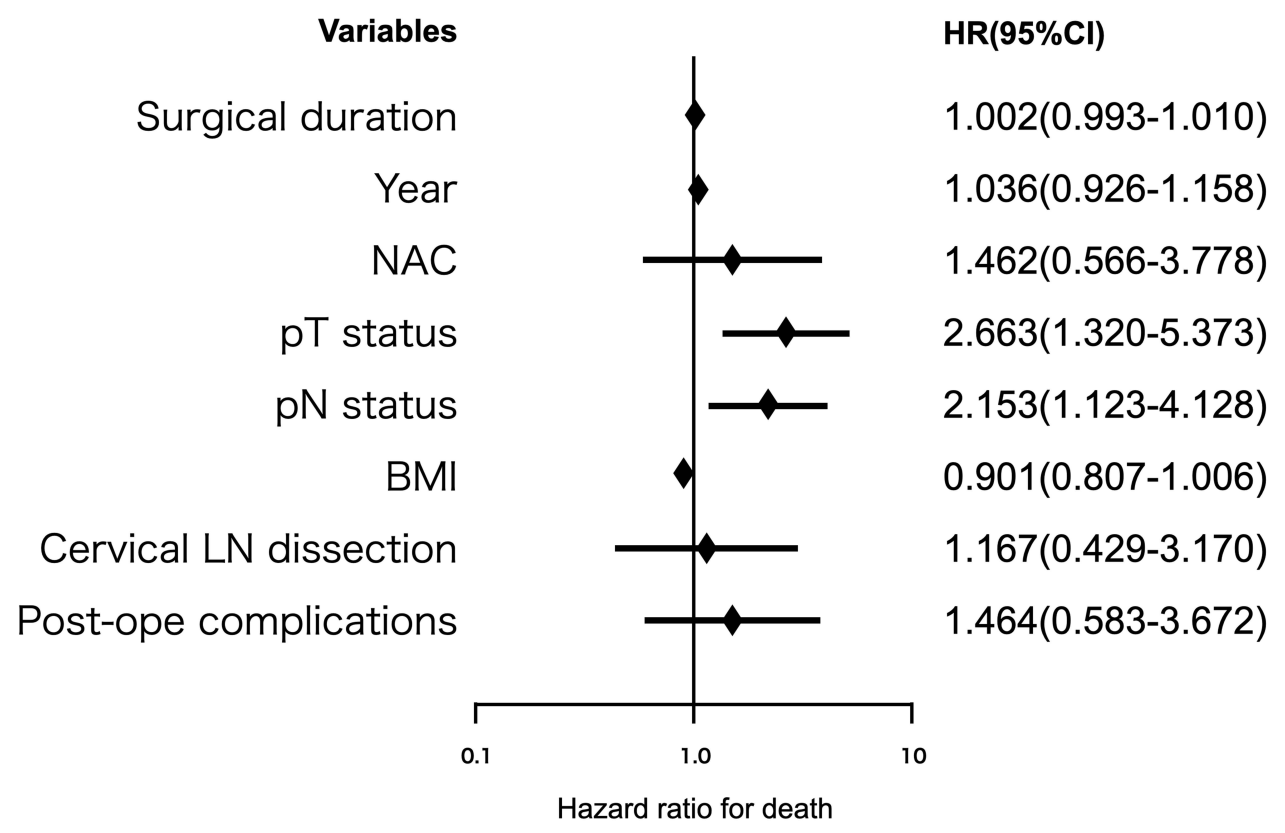

Figure 2 Factors associated with overall survival. Cox proportional hazard model were used to find influence variables on patient survival. In forest plots, hazard ratio (HR) and $95 \%$ confidence interval $(\mathrm{Cl})$ are described on each variable.

Abbreviations: NAC, neo-adjuvant chemotherapy; pT, pathological grades of depth of tumor invasion; pN, pathological grades of lymph node metastasis; BMI, body mass index; LN, lymph node. 


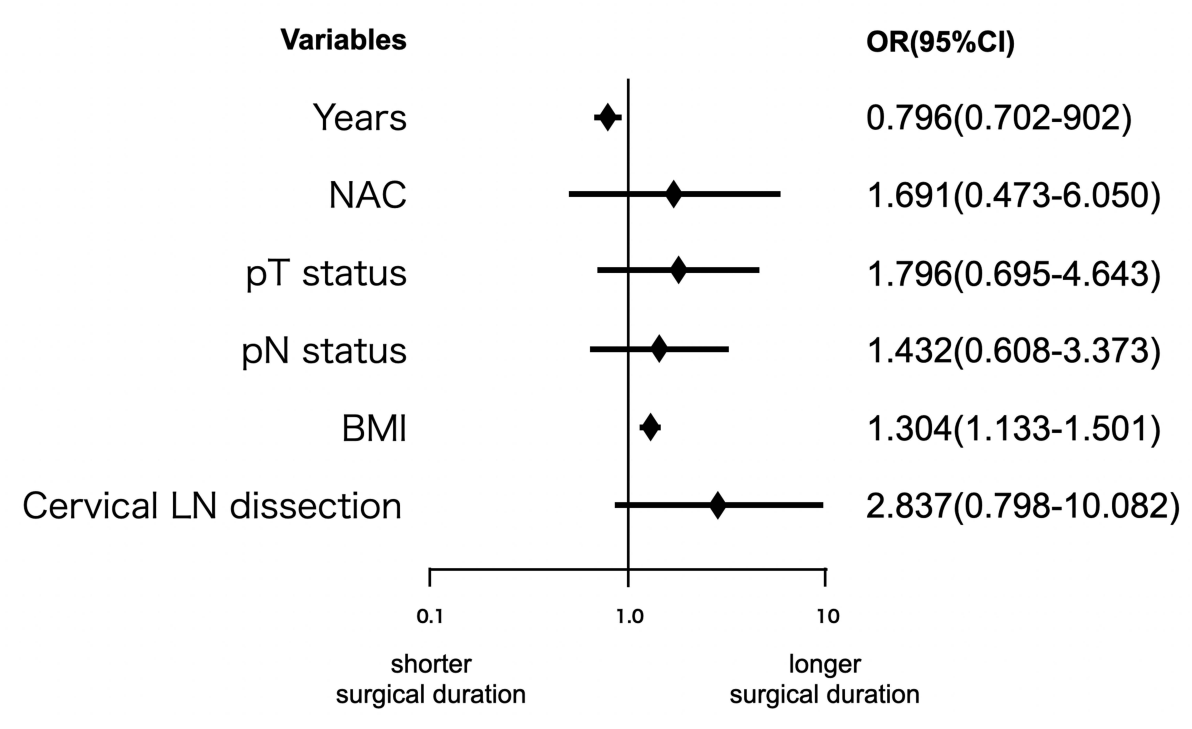

Figure 3 Factors associated with surgical duration. Logistic regression analysis was used to find influence variables on surgical duration of Ivor-Lewis approach. In forest plots, odds ratio (OR) and $95 \%$ confidence interval $(\mathrm{Cl})$ are described on each variable.

Abbreviations: NAC, neo-adjuvant chemotherapy; pT, pathological grades of depth of tumor invasion; pN, pathological grades of lymph node metastasis; BMI, body mass index; LN, lymph node.

showed that surgical duration was becoming shorter over the years (OR 0.796, $P=0.000)$. This is probably due to the continuous refinement of the surgeon's technique over the years.

We found that surgical duration did not affect patient survival in our series of Ivor-Lewis esophagectomy. Of course, different surgical techniques will result in different surgical durations. New surgical techniques have been introduced over the years, and good procedures become standard techniques with continual advancements. Minimally invasive surgery can be done with a small skin incision and fewer scars, but require extensive practice for technical improvement are necessary in order to shorten surgical duration. A study enrolling 9584 patients using the Nationwide Database in Japan used propensity score matching in patients undergoing open esophagectomy or minimally invasive esophagectomy and concluded that minimally invasive surgery had high rates of recurrent laryngeal nerve palsy and re-operation, small amounts of bleeding, and lower rates of wound infection and atelectasis. ${ }^{14}$ Surgical duration, although significantly prolonged in the minimally invasive esophagectomy group, did not affect 30-day mortality. The superiority of minimum invasive surgery remains controversial, but a comparative study on long-term survival is currently underway in Japan (JCOG1409). The surgical approach in our study was limited to open esophagectomy, which differed significantly from thoracoscopic surgery in terms of surgical duration, and we could not demonstrate any correlation between shorter surgical duration and better OS. However, it would also be true that faster surgical procedures lead to shorter anesthesia times, increasing the efficiency of operating room utilization and decreasing operation costs, and can increase overall surgical productivity.

The present study has several limitations that should be acknowledged. First, this was a retrospective study conducted in a single institution without randomization, with a small study cohort that was collected over a 10-year period. Second, the surgical procedure was limited to the open approach, and whether the study findings can be extended to thoracoscopic esophagectomy remains unclear. Third, NAC as a therapeutic option before surgery was initiated in 2010 at our institution. Therefore, 39 patients included in this study before 2009 did not receive preoperative chemotherapy in spite of possible candidate of NAC. Finally, the survival time analysis unfortunately included 25 (19\%) patients with an observation period of less than three years due to the short follow-up time after the esophagectomy.

\section{Conclusion}

According to our analysis of patients who underwent the same surgical approach by the same surgeon, we did not observe any benefit of a shorter surgical duration on patient survival. However, we believe that it is worthwhile 
for surgeons to shorten the surgical duration by refining their skills and performing their best.

\section{Acknowledgments}

[The authors would like to thank Enago (Crimson Interactive Japan Co.,Ltd, Japan) for the English language review.

\section{Disclosure}

The authors report no conflicts of interest in this work.

\section{References}

1. Ball CG, Pitt HA, Kilbane ME, et al. Peri-operative blood transfusion and operative time are quality indicators for pancreatoduodenectomy. $H P B$ (Oxford). 2010;12:465-471. doi:10.1111/j.1477-2574.2010.00209.x

2. Procter LD, Davenport DL, Bernard AC, et al. General surgical operative duration is associated with increased risk-adjusted infectious complication rates and length of hospital stay. $\mathrm{J} \mathrm{Am} \mathrm{Coll} \mathrm{Surg.}$ 2010;210:60-65.e1-2. doi:10.1016/j.jamcollsurg.2009.09.034

3. Yang CK, Teng A, Lee DY, et al. Pulmonary complications after major abdominal surgery: National Surgical Quality Improvement Program analysis. J Surg Res. 2015;198:441-449. doi:10.1016/j.jss.2015.03.028

4. Robin LH, Gospodarowicz MK, Wittekind C, editors. The TNM Classification of Malignant Tumors. 7th ed. Oxford: Wiley-Blackwell ; 2011.

5. Kuwano H, Nishimura Y, Oyama T, et al. Guidelines for diagnosis and treatment of carcinoma of the esophagus April 2012 edited by the Japan Esophageal Society. Esophagus. 2015;12:1-30. doi:10.1007/ s10388-014-0465-1

6. Dindo D, Demartines N, Clavien P. Classification of surgical complications a new proposal with evaluation in a cohort of 6336 patients and results of a survey. Ann Surg. 2004;240:205-213. doi:10.1097/01. sla.0000133083.54934.ae
7. Ando $\mathrm{N}$, Kato $\mathrm{H}$, Iwaki $\mathrm{H}$, et al. A randomized trial comparing postoperative adjuvant chemotherapy with cisplatin and 5-fluorouracil versus preoperative chemotherapy for localized advanced squamous cell carcinoma of the thoracic esophagus (JCOG9907). Ann Surg Oncol. 2012;19:68-74. doi:10.1245/s10434011-2049-9

8. Kobayashi M, Koh M, Irinoda T, et al. Stroke volume variation as a predictor of intravascular volume depression and possible hypotension during the early postoperative period after esophagectomy. Ann Surg Oncol. 2009;16:1371-1377. doi:10.1245/s10434-008-0139-0

9. Kobayashi M, Irinoda T, Akiyama Y, et al. Effect of a selective neutrophil elastase inhibitor on early recovery from body water imbalance after transthoracic esophagectomy. Dis Esophagus. 2010;23:565-571. doi:10.1111/j.1442-2050.2010.01053.x

10. Ando N, Iizuka T, Ide H, et al. Surgery plus chemotherapy compared with surgery alone for localized squamous cell carcinoma of the thoracic esophagus: a Japan Clinical Oncology Group Study. JCOG 9204. J Clin Oncol. 2003;21:4592-4596. doi:10.1200/ JCO.2003.12.095

11. Valsangkar N, Salfity HVN, Timsina L, et al. Operative time in esophagectomy: does it affect outcomes? Surgery. 2018;164 (4):866-871. doi:10.1016/j.surg.2018.06.020

12. van der Schaaf MK, Tilanus HW, van Lanschot JJ, et al. The influence of preoperative weight loss on the postoperative course after esophageal cancer resection. $J$ Thorac Cardiovasc Surg. 2014;147:490-495. doi:10.1016/j.jtcvs.2013.07.072

13. Zhang SS, Yang H, Luo KJ, et al. The impact of body mass index on complication and survival in resected oesophageal cancer: a clinical-based cohort and meta-analysis. $\mathrm{Br} J$ Cancer. 2013;109:2894-2903. doi:10.1038/bjc.2013.666

14. Takeuchi H, Miata H, Ozawa S, et al. Comparison of short-term outcomes between open and minimally invasive esophagectomy for esophageal cancer using a Nationwide Database in Japan. Ann Surg Oncol. 2017;24:1821-1827. doi:10.1245/s10434-017-5808-4
Open Access Surgery

\section{Publish your work in this journal}

Open Access Surgery is an international, peer-reviewed, open access journal that focuses on all aspects of surgical procedures and interventions. Patient care around the peri-operative period and patient outcomes post-surgery are key topics for the journal. All grades of surgery from minor cosmetic interventions to major surgical procedures are covered. Novel techniques and the utilization of new

Submit your manuscript here: https://www.dovepress.com/open-access-surgery-journa instruments and materials, including implants and prostheses that optimize outcomes constitute major areas of interest. The manuscript management system is completely online and includes a very quick and fair peer-review system, which is all easy to use. Visit

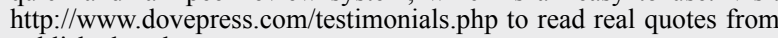
published authors. 